\section{Linguistica ancilla theologiae}

\section{L'intérêt de la linguistique cognitive pour la théologie fondamentale}

J'ai décoưvert la métaphore selon la 'linguistique cognitive' à l'occasion de ma collaboration avec K. Feyaerts, professeur de linguistique, à la conférence $\mathrm{LAUD}^{1}$ de 1997 , organisée autour du thème 'Métaphore et religion'. Puisque 'la métaphore' est devenue un thème central de l'épistémologie théologique récente, il m'a semblé utile d'examiner les présupposés, la méthode et les champs d'application de la 'linguistique cognitive'2. La raison de mon intérêt sera évidente pour chaque théologien: quoique nous soyons de plus en plus attentifs à l'innommable de Dieu, et que dans ce contexte nous problématisions la portée cognitive et le poids du langage religieux, en particulier du discours sur Dieu, nous continuons néanmoins en tant que croyants à parler à Dieu et à parler de Dieu, pour tenter d'exprimer le cour de nos croyances. Les paroles et les écrits de la linguistique cognitive sur la métaphore sont une source qui permet de reévaluer la cognitivité du langage religieux à partir d'une perspective théologico-épistémologique. Une telle réflexion interdisciplinaire avait déjà été amorcée dans la contribution LAUD mentionnée ci-dessus ${ }^{3}$. Je voudrais ici prolonger cet essai.

${ }^{1}$ LAUD = Linguistic Agency University of Duisburg. Les actes du colloque ont paru dans R. BISSCHOPS et J. Francis, Metaphor, Canon and Community (coll. Religions and Discourse, 1), Berne-Berlin-Bruxelles, 1999; et L. BoEve et K. FEYAERTs (eds.), Metaphor and God-talk (coll. Religions and Discourse, 2), K. FEYAERTS (eds.), Metaphor

${ }^{2}$ La théorie de la métaphore de la linguistique cognitive, ici en particulier la sémantique cognitive, a été développée par des auteurs anglo-saxons comme George Lakoff, Mark Johnson, Mark Turner e.a. Une courte comparaison des quatre grandes théories de la métaphore, respectivement rhétorique (Aristote, Quintilien), interactioniste (Richards, Black, Ricour), pragmatique (Speech Act Theory de Austin Searle) et cognitive, est présentée dans l'introduction de $L$. BOEVE et $K$. FEYAERTS, Op. cit., p. $7-14$.

3 IDEM, Religious Metaphors in a Postmodern Context. Transverse Links between Apophatical Theology and Cognitive Semantics, in Ibidem, p. 153-185.
La perspective n'est ni philologique, ni philosophique, ni même exégétique, mais fondamentalement théologique. Une des meilleures définitions de la théologie chrétienne n'est-elle pas celle d'Anselme de Cantorbéry: fides quaerens intellectum, «la foi cherchant la compréhension»? Enracinés dans leurs traditions de foi particulières, les théologiens cherchent à éclairer l'attitude (fides qua) et le contenu (fides quae) de la foi. À une époque lointaine déjà, et certainement depuis l'insertion de la foi chrétienne dans le contexte hellénistique, les théologiens ont emprunté à la culture de leur temps une nouvelle terminologie et les structures de pensée les plus adéquates pour poursuivre cette tâche. Très souvent ils recouraient au vocabulaire et aux schèmes philosophiques comme fruits de la conscience criticoréflexive de leur contexte. Ce procédé se reflète dans un autre adage théologique classique, philosophia ancilla theologiae, «la philosophie est la servante de la théologie». L'histoire de la théologie le montre: chaque fois que les contextes historiques et la conscience critique de ceux-ci se transforment, la théologie est obligée de se recontextualiser en cherchant de nouvelles connexions avec la philosophie contemporaine, et notamment depuis l'essor des sciences, avec les sciences humaines, culturelles et sociales ${ }^{4}$. C'est dans cette perspective que la théologie fondamentale s'intéresse à la méthode et au champ de la linguistique cognitive. D'où la question: la réflexivité de la linguistique pourrait-elle contribuer à une recontextualisation de l'épistémologie théologique: Linguistica ancilla theologiae?

\section{LE LANGAGE RELIGIEUX ET LA THEOLOGIE} DANS UN CONTEXTE POST-MODERNE

La recontextualisation n'est pas 1'apanage de la théologie, mais une structure de base de la religion en tant que telle, en particulier du christianisme, et donc aussi de la théologie. En effet, les traditions religieuses ne sont pas des entités statiques, mais des réalités prises dans une dynamique de réception et de (re)construction. Le langage religieux est ainsi recontextualisé et commence à 'muter' par le jeu

${ }^{4}$ L. BoEvE, Onderbroken traditie. Heeft het christelijk verhaal nog toekomst?, Kapellen, 1999 et L. BOEVE, La conscience critique dans la condition postmoderne. de nouvelles possibilités pour la théologie?, in Nouvelle revue théologique, t. 132, 2000 , p. 68-86. 
interactif des expériences de la nouveauté/altérité d'une part, et de l'interprétation traditionnelle d'autre part.

Dans la théologie chrétienne, ce processus dynamique est activé et légitimé par la conscience religieuse critique du deus semper major. Ceci explique que le langage religieux, tout en témoignant de Dieu, n'est pas en état de décrire ou de saisir Dieu définitivement. Cette conscience s'explicite particulièrement dans les traditions de la théologie apophatique, affirmant qu'en fin de compte on honore davantage Dieu en niant les images et les concepts qui le concernent, qu'en les affirmant. La théologie oubliant ou refusant de tenir compte de la conscience apophatique aboutit à l'idolâtrie. II faut mentionner que Denys l'Aréopagite, père de ladite théologie apophatique, est un interlocuteur majeur de Thomas d'Aquin dans la Summa Theologiae.

Après l'abandon des 'grands récits modernes' de la connaissance et l'émancipation, dans ce que certains appellent le contexte postmoderne, une telle conscience religieuse critique est entretenue par la sensibilité contemporaine à l'égard de la contextualité, de la particularité, de l'hétérogénéité et de l'altérité 5 . Dans la pensée contemporaine, ce sont par excellence les philosophies de la différence qui transforment la conscience contextuelle en objet de réflexion. À travers de telles structures réflexives, la théologie est appelée à se recontextualiser, et ainsi à se transformer en un discours sur Dieu qui ne prend pas appui sur la présomption d'être fondé sur un socle universellement métaphysique et qui légitimerait ses prétentions à la vérité. Grâce à la recontextualisation, la théologie apprendrait à percevoir son discours sur Dieu comme radicalement contextuel, particulier et contingent - étant motivé théologiquement par son engagement à l'égard de l'altérité divine. Dieu, tout d'abord, n'est pas compris en termes d'identité, mais d'hétérogénéité. Dieu est l'Autre radical et se soustrait aux tentatives d'identification, surtout sous forme de conceptualisation. Dieu ne peut pas être saisi complètement par le discours théologique et, en outre, toute tentative de ce genre est soumise à une pression critique, soulignant le danger presque inévitable de l'idolâtrie, qui constitue le reflet divinisé de notre propre perspective limitée.

${ }^{5}$ L. BoEve, Postmodernism and Negative Theology. The A/theology of the 'Open Narrative', in Bijdragen. Tijdschrift voor filosofie en theologie, t. 58, 1997, p. 407425 .
Quoi qu'il en soit, témoigner de Dieu en respectant son altérité irréductible implique un discours, un langage. Il n'y a pas d'apophase sans cataphase, pas de négation sans affirmation. En outre, au sein de la négation apophatique de ce qui se donne cataphatiquement, le sens de ce qui est nié n'est pas effacé, mais transféré 'hyperphatiquement' dans un discours évocateur au-delà de la cataphase et de l'apophase, au-delà de l'affirmation (ou de la prédication) et de la négation. En d'autres termes, bien que le langage religieux, et en particulier le discours sur Dieu, soit loin d'atteindre Dieu à travers la référence à Lui, il n'est pas arbitraire, car il trouve son origine dans une tradition particulière qui n'est pas fondée métaphysiquement. Et c'est précisément grâce à ces racines traditionnelles que ce discours peut évoquer Dieu 'hyperphatiquement'6.

C'est à partir d'une perspective théologico-épistémologique que surgit la question de la portée cognitive du langage religieux. Dans notre ère 'post-ontothéologique', épistémologiquement, la cognitivité ne semble plus vouée à la correspondance (l'ordre logique reflétant l'ordre ontologique) ${ }^{7}$, ni à la même cohérence (la cognitivité interne

6 Le terme 'hyperphase' est un néologisme, créé par analogie avec 'hyperphatos', lui-même dérivé de 'hyper-phèmi', signifiant 'au-delà du langage, ineffable' (H.G. LIDDELL et R. SCOTT, A Greek-English Lexicon. With a Supplement. Revised and Augmented by H. STUART JONES with the Assistance of R. MCKENZIE, Oxford, 1968, p. 1870). L'hyperphase est également (cfr p. 1864 et 1869) une alternative pour le mot 'hyp ' ', : 'h ' ' gique de 'hyperphase', l'on se fonde sur la conscience de la particularité du discours sur Dieu: bien que le théologien nie apophatiquement ce qui se dit de Dieu dans la cataphase, cette négation n'est pourtant pas un effacement, mais plutôt, pour ainsi dire, une biffure: les mots couchés sur papier et qui sont biffés, restent néanmoins lisibles, et forment ainsi une nouvelle unité sémantique qui ne saurait être réduite à une affirmation ou à une simple négation. D'ailleurs, la biffure est impossible sans ces mots, dont le fonctionnement à travers la négation est renouvelé dans le but de renvoyer à un référent insaisissable. Jean-Luc Marion par exemple, dans son Dieu une quelconque position onto-théologique, biffe de manière conséquente le mot Dieu - suivant la linguistique cognitive, c'est le concept de 'Dieu' qui est impliqué afin de se référer à celui qui échappe à la référence. De cette façon, le discours sur Dieu est privé de son potentiel d'affirmer des prédicats de Dieu. Cfr J.-L. MARION, Dieu sans l'être, Paris, 1982, p. 72: «De Dieu, admettons clairement que nous ne pouvons le penser que sous la figure de l'impensable, mais d'un impensable qui outrepasse aussi bien ce que nous ne pouvons pas penser que ce que nous pouvons penser; car ce que je ne puis penser, cela relève encore de ma pensée, et donc me reste penchenrise comme l'aura de son advenue, la gloire de son insistance, l'éclat de son retrait».

7 C'est dans cette perspective que Thomas d'Aquin a formule l'analogie, en accentuant en même temps la continuité (ontologique) et la discontinuité (épistémologique) entre le langage concernant le monde et le langage qui concerne Dieu. Voir note 25. 
sans aucune structure référentielle), mais elle se découvre dans la manière selon laquelle un récit religieux s'organise, aussi bien au niveau du contenu que structurellement, afin d'exprimer la relation au Dieu innommable (la cognitivité saisie à travers une structure référentielle irrémédiablement rompue).

Comment donc - étant donné cette conscience apophatique - le discours sur Dieu et, plus généralement, le langage religieux disentils quelque chose sur Dieu et sur la réalité de la vie religieuse? Et quelle sorte de cognitivité est impliquée dans pareil discours? La théorie de la métaphore, telle qu'elle est formulée dans la linguistique cognitive, donne à penser et offre des structures réflexives qui permettent de recontextualiser l'épistémologie théologique. Nous esquisserons d'abord les lignes principales de cette approche, y compris les définitions du sens et de la vérité, et nous rendrons compte de la place prépondérante accordée à la métaphore. Ensuite, nous formulerons en cinq thèses les résultats théologiques qui peuvent surgir de la rencontre avec la linguistique cognitive.

\section{COGNITIVITÉ ET MÉTAPHORE:}

\section{QUELQUES PERPECTIVES DE LA LINGUISTIQUE COGNITIVE}

\section{Cognitivité dans la linguistique cognitive}

Pour la linguistique cognitive ${ }^{8}$, la cognitivité réside dans la structure conceptuelle qui sous-tend notre approche de la réalité, et non dans une relation supposée entre les ordres logique et ontologique, comme dans l'approche objectiviste. La connaissance ne reflète pas la réalité, au contraire, elle est localisée au sein même de l'organisation de notre approche de la réalité; elle relève de la compréhension, c'est-à-dire du 'profilage', de la 'caté-

8 Pour une bibliographie pertinente sur la linguistique cognitive, voir L. BoEve et K. FEYAERTS, Religious Metaphors in a Postmodern Context (n. 3). Pour un bref aperçu historique, voir E. SwEETSER, From Etymology to Pragmatics. Metaphorical and Cultural Aspects of Semantic Structure, Cambridge, 1990, p. 16 et suivantes.

9 Le 'profilage' consiste à qualifier en mettant en exergue, en soulignant certaines caractéristiques, ce qui a pour conséquence que d'autres traits apparaissent seulement à l'arrière-plan. Cfr G. LAKofF et M. JoHNSON, Metaphors We Live By, Chicago, 1980, p. 163. Ci-dessous, nous donnons la traduction de quelques textes essentiels de cet ouvrage. gorisation'10 et de la 'projection'; elle relève du raisonnement par images ${ }^{11}$. La signification n'est pas une donnée objective, mais elle consiste en ce qui est significatif pour nos vies, suivant nos expériences quotidiennes. C'est dire que les structures conceptuelles sont fondées dans la réalité par expérience et que, en même temps, elles déterminent notre interaction sociale avec la réalité ${ }^{12}$. C'est pourquoi la vérité ne relève pas simplement de la correspondance entre une affirmation et un état des choses, mais elle se construit par le biais de notre compréhension à la fois de ce qui est dit et de ce qui est en jeu. Pourtant, puisque les structures conceptuelles peuvent différer suivant le moment, le lieu et la culture, les prétentions à la vérité ne sont pas universellement valides, ni arbitraires ni subjectivistes, mais dépendent du contexte culturel qui les soutient ${ }^{13}$.

En général, l'on pourrait arguer que la linguistique cognitive prend en compte beaucoup d'éléments que nous avons identifiés dans la conscience critique contemporaine (il est vrai toutefois que le degré de concordance sera différent pour chaque sémanticien cognitif). En effet, dans Metaphors We Live By, George Lakoff et Mark Johnson, deux des plus éminents propagateurs de la linguistique cognitive, prétendent «qu'il n'y a rien de vraiment nouveau dans [leur] acception de la vérité. Celle-ci inclut certains acquis principaux de la tradition phénoménologique, comme le rejet du 'fondationalisme' épistémologique, l'accent mis sur la centralité du corps dans la structuration de notre expérience, et l'importance de cette structure dans la compréhension. [Leur] manière de voir s'accorde avec quelques-uns des élé-

${ }^{10}$ La 'catégorisation' est la structuration à l'aide de ressemblances de famille. Dans ce contexte, les catégories-prédicats ne sont pas des propriétés intrinsèques de l'objet, mais des propriétés interactionnelles, «qui signifient seulement en relation avec le fonctionnement humain» (Ibid., p. 164).

${ }_{11}$ La projection organise en considérant une chose en termes d'une autre - c'est ici que commence la théorie de la métaphore selon la linguistique cognitive.

${ }_{12}^{2}$ Voir Ibid., p. 180-181: «Du point de vue expérimentaliste, notre système conceptuel émerge de notre fonctionnement constamment fructueux dans notre environnement physique et culturel. Nos catégories de l'expérience et la dimension à partir de laquelle elles sont construites n'ont pas seulement émergé de notre expérience, mais elles sont constamment testées par le fonctionnement toujours fructueux de tous les membres de notre culture».

13. «Des gens avec des systèmes conceptuels très différents des nôtres peuvent comprendre le monde d'une autre façon que nous. Ainsi, ils peuvent avoir un ensemble de vérités très différent du nôtre et même des critères différents pour la vérité et la réalité» (Ibid., p. 181). 
ments-clés de la philosophie du dernier Wittgenstein: la catégorisation en termes de ressemblance-famille, le rejet de la théorie de la signification-reflet, le rejet de la théorie de la signification-pierre de construction, et l'emphase sur la signification mise en relation avec le contexte et le système conceptuel de l'individu» ${ }^{14}$.

En ce qui concerne la relation entre signification et langage, il faudrait faire une distinction entre la structure conceptuelle, la structure sémantique et l'expression linguistique. La signification, comme il a été dit plus haut, doit être identifiée aux structures conceptuelles riches dans l'esprit du locuteur. En aucune manière, les structures conceptuelles ne peuvent être saisies complètement dans les structures sémantiques conventionnelles (la signification conventionnelle) impliquées dans les expressions linguistiques. L'expression linguistique du fils adorant sa mère, " $J$ 'ai la meilleure maman du monde", porte un surplus de sens, contenu dans la structure conceptuelle MAMAN qu'il a dans l'esprit, et qui n'est pas totalement évoqué dans la valeur sémantique conventionnalisée de l'expression "maman". Or, c'est par le biais de cette structure conceptuelle que le fils gère la réalité, c'est-àdire qu'il la comprend, qu'il en fait l'expérience, qu'il agit en elle. La différence entre structure conceptuelle, structure sémantique, et expression linguistique est également représentée de façon formelle. Par convention, les petites lettres capitales indiquent la structure conceptuelle MAMAN, tandis que les guillemets de la citation représentent la structure sémantique "maman". Les italiques maman signalent l'expression linguistique entière.

\section{Métaphore}

La théorie sémantico-cognitive de la métaphore, interprétée comme le fait de raisonner en images, est d'une importance capitale pour la formation et le fonctionnement des structures conceptuelles. Dans ce cadre, la métaphore n'est plus perçue comme un instrument linguistique, comme 'du simple langage' 15 , mais principalement comme un

${ }_{15}^{14}$ Ibid., p. 181-182.

15 Suivant l'acception classique, la métaphore est considérée comme l'emploi différent d'un mot afin de «produire un changement de sens fondé sur les similarités entre les choses [comparées]» (M. JoHNSON, Introduction: Metaphor in the entre les choses [comparees]» (M. JOHNSON, Introduction: Metaphor in the
Philosophical Tradition, dans M. JoHNSON [ed.], Philosophical Perspectives on Metaphor, Minneapolis, 1981, p. 6). phénomène conceptuel toujours présent, activé dans les expressions linguistiques. La métaphore est donc définie comme la superposition de deux concepts appartenant à des domaines différents de la connaissance. Le résultat de la superposition est qu'un concept (la cible) est structuré (compris) dans les termes de l'autre (la source). Un exemple classique est LA DISCUSSION, C'EST LA GUERRE ${ }^{16}$. Les schémas conceptuels activés dans la compréhension du domaine de la GUERRE sont projetés dans le domaine de la DISCUSSION. Les expressions linguistiques qui actualisent la métaphore sont, par exemple, défendre une opinion, attaquer la position de l'adversaire, gagner ou perdre dans une discussion. En examinant de plus près la pratique quotidienne, on doit reconnaître qu'une partie majeure de notre compréhension, et donc de notre langage, est structurée métaphoriquement. La compréhension, la signification et le raisonnement en termes d'image vont de pair ici, et ils ont des implications immédiates dans la manière d'expérimenter le 'monde', de l'interpréter et de l'affronter. Il est évident qu'une structuration de la DISCUSSION en termes de GUERRE diffère considérablement de la superposition: LA DISCUSSION, C'EST LA DANSE. Une grande partie de ce qui est communément considéré comme du langage 'littéral' résulte en fait des actualisations de métaphores conventionnelles: par exemple "J'ai le moral bas" actualise la métaphore HEUREUX/PLUS/BON EST HAUT; TRISTE/MOINS/MAUVAIS EST BAS: des sentiments, des attitudes, des évaluations positifs et négatifs sont souvent structurés à l'aide de cette métaphore d'orientation, qui est fondée physiquement (quand on verse de l'eau dans un verre par exemple, l'eau monte) et acceptée culturellement.

J'ajoute trois observations. (1) Beaucoup de concepts, surtout abstraits, comme TEMPS, VIE, AMOUR, DIEU sont essentiellement structurés métaphoriquement. Ainsi: UNE THÉORIE EST UN BÂTIMENT, LE TEMPS EST DE L'ARGENT, LA VIE EST UN VOYAGE, L'AMOUR EST UN PARTENARIAT, DIEU EST PÈRE ${ }^{17}$. Puisqu'ils ne sont pas autonomes au niveau sémantique, ces concepts sont métaphoriques; ils sont compris au moyen de structures appartenant à d'autres domaines de la connaissance. (2) En plus, riches et complexes, ils sont souvent structurés selon plusieurs domaines à la fois. L'AMOUR n'est pas seulement un

16 Tous les exemples, sauf indication explicite, sont pris de G. LAKOFF et M. JoHnson, Op. cit., passim.

${ }_{17}$ Cfr notre exemple. 
PARTENARIAT (les amoureux investissent dans leur relation), il est également UNE FORCE PHYSIQUE (il y a de l'électricité entre eux), ou un PATIENT (ils avaient une relation maladive), de la FOLIE (il est fou d'elle), de la MAGIE (elle est envoûtante), la GUERRE (elle a gagné son amour; il a fait beaucoup de conquêtes rapides). Chaque métaphore met en exergue un aspect particulier de nos expériences et de nos structures de pensée concernant l'amour. (3) Enfin, dans une métaphore conventionnelle, chaque élément du domaine source n'est pas superposé à la cible. Seules des structures pertinentes pour nos expériences et interprétations de la réalité véhiculées par le concept cible sont utilisées ${ }^{18}$.

Il est à noter que les processus de superposition métaphorique sont fondés par expérience; ils sont motivés par la totalité de l'expérience humaine ${ }^{19}$. En d'autres termes, des domaines sémantiquement non autonomes sont ultimement structurés métaphoriquement dans les termes de domaines qui sont autonomes sur le plan sémantique, c'est-à-dire des domaines qui reçoivent leur structure de notre engagement concret avec la réalité physique et culturelle (HAUT/BAS, CHOSE). Ils sont également fondés culturellement: les sensibilités culturelles n'ont pas seulement pour fonction de motiver le processus de superposition (LE TEMPS EST DE L'ARGENT), mais elles influencent également les structures conceptuelles, qui font figure de domaines sources destinés à être superposés aux cibles à structurer ${ }^{20}$.

${ }^{18}$ LE TEMPS EST DE L'ARGENT ne renvoie pas nécessairement au fait que l'argent serait représenté concrètement, à savoir en pièces ou en billets.

19 Voir G. LAKOFF et M. JOHNSON, Op. cit., p. 224-225: «notre système conceptuel a une structure [...] en termes de corps [Gestalts] expérimentaux. La structure de ces corps est loin d'être arbitraire. En effet, les dimensions qui caractérisent la structure de ces corps émergent de manière naturelle de notre expérience. Ceci ne veut pas dire qu'il faille exclure la possibilité que la signification d'une chose soit basée sur des genres d'expériences que $\mathrm{j}$ 'ai eues et que vous n'avez pas eues et que, ainsi, sur des genres d'expériences que j'ai eues et que vous n'avez pas eues et que, ainsi,
je ne sois pas capable de vous communiquer entièrement et adéquatement la signification en question. Toutefois, la métaphore fournit une façon de communiquer partiellement les expériences non partagées, et c'est la structure naturelle de notre expérience qui le rend possible».

${ }^{20}$ Voir L. BoeVE et K. FeyaerTs, Religious Metaphors in a Postmodern Context (n. 1), p. 173, avec une référence à THE GREAT CHAIN OF BEING (G. LAKOFF et M. TURNER, More than Cool Reason. A Field Guide to Poetic Metaphor, Chicago, 1989 , p. 166), et ANGER IS HEAT (D. GEERAERTS et S. GRONDELAERS, Looking Back at Anger: Cultural Traditions and metaphorical Patterns, dans J.R. TAYLOR et R.E. MCLAURY (eds.), Language and the Cognitive Construal of the World, Berlin, 1995, p. 153-179).
La métaphore DIEU EST PÈRE, par exemple, née dans un contexte patriarcal, sera structurée différemment dans un contexte postpatriarcal.

Ce fondement par expérience est une donnée dynamique, liée aux réalisations antérieures de métaphores conventionnelles dans la 'vie réelle', comme par exemple dans les institutions et les pratiques sociales, lors des rituels, dans les récits et les mythes, etc. ${ }^{21}$. «Les bases expérimentales et les réalisations métaphoriques sont l'envers et l'endroit d'une même médaille: elles sont des corrélations de l'expérience réelle qui ont la même structure que les corrélations des métaphores. Cependant les bases expérimentales précèdent les superpositions métaphoriques conventionnelles, elles les fondent et leur attribuent un sens, tandis que les réalisations suivent et se voient attribuer un sens via les métaphores conventionnelles. D'ailleurs, les réalisations d'une métaphore par une génération donnée, peuvent faire partie de la base expérimentale de la même métaphore utilisée par la génération suivante» 22 .

En guise de conclusion: une telle analyse «de l'activité cognitive dans les processus de métaphorisation» souligne «la véritable signification épistémologique [...] de la métaphore, à savoir le fait qu'elle fonctionne comme un instrument dans la réorganisation de nos structures perceptives et/ou conceptuelles ${ }^{23}$. Donc, des métaphores ont bel et bien un statut cognitif. Elles ne sont pas cognitivement réductibles à un langage littéral. La dimension 'comparative' de la métaphore n'est plus inférieure a priori à la dimension 'constative' du langage littéral; en réalité, souvent le soi-disant 'constatif' implique du 'comparatif': «Nous nous heurtons au monde, non de manière passive, mais par le biais d'actes de projection influencés par nos intérêts, nos objectifs, nos valeurs, nos croyances et notre langue. Étant donné que notre monde est une construction imaginative,

${ }^{21}$ Voir G. LAKOFF, The Contemporary Theory of Metaphor, dans A. ORTONY (ed.), Metaphor and Thought, Cambridge, $1993^{2}$, p. 244: une métaphore se réalise «quand quelque chose de réel s'ouvre à la compréhension et devient même évident, naturel, par le biais d'une métaphore conventionnelle qui le dote d'une structure. Ainsi, beaucoup de 'réel' dans la société ou dans l'expérience de l'individu devient signifiant via la structuration qu'engendre une métaphore conventionnelle».

${ }_{22}$ G. LAKOFF, The Contemporary Theory of Metaphor, dans A. ORTONY (ed.), ${ }^{22}$ G. LAKOFF, The Contemporary Theory of Metaphor
Metaphor and Thought, Cambridge, 19932, p. 202-251.

${ }^{23}$ M. JoHnson, Introduction: Metaphor in the Philosophical Tradition, dans M. JoHNSON (ed.), Philosophical Perspectives on Metaphor, Minneapolis, 1981, p. 31. 
imbue de valeurs, les métaphores qui changent nos structures conceptuelles (véhiculées par des métaphores plus anciennes) changeront également la manière dont nous vivons les choses» ${ }^{24}$.

\section{L'ÉPISTÉMOLOGIE THÉOLOGIQUE ET LA THÉORIE SÉMANTICO- COGNITIVE DE LA MÉTAPHORE}

Compte tenu de la conscience apophatique de la théologie, nous pouvons formuler en cinq thèses les résultats théologiques possibles de la rencontre intensive avec la linguistique cognitive, et plus particulièrement avec la théorie de la métaphore. Dans les quatre premières thèses, nous esquisserons d'abord l'apport de la linguistique cognitive, et ensuite nous évoquerons les questions ou discussions théologiques que cette rencontre pourrait engendrer. Dans la cinquième thèse, nous réfléchirons aux conditions d'une incorporation théologique de quelques traits de la linguistique cognitive au sein de l'épistémologie théologique.

\section{Le discours sur Dieu est en principe métaphorique}

Traduire la conscience apophatique, critique et herméneutique de la théologie dans le langage sémantico-cognitif souligne le caractère strictement métaphorique du discours sur Dieu. Le concept de DIEU, comme tous les concepts abstraits, est non autonome sur le plan sémantique - et ceci de la façon la plus radicale qui soit. 'Dieu' n'évoque pas en lui-même des schèmes de connaissance, sans interférence d'autres domaines. C'est pourquoi tout le savoir sur Dieu est constitutivement métaphorique.

Certes, le fait n'est pas nouveau pour la plupart des théologiens. Toutefois, affirmer que le discours sur Dieu est absolument métaphorique soulève des questions à propos de l'idée d'un mode de discours analogique sur Dieu, qui serait qualitativement différent du mode métaphorique et posséderait son propre statut cognitif. Prononcer les phrases 'Dieu est bon' ou 'Dieu est sage' (ce qu'on appelle les perfections simples) implique alors une autre qualité de la cognitivité que celle qu'on trouve dans la phrase 'Dieu est un rocher'. Selon

${ }^{24}$ Ibidem, p. 41.
Thomas d'Aquin, les analogies sont distinctes en cognitivité, car ces perfections simples (être bon, sage etc.), puisqu'elles sont fondées sur l'analogia entis ou analogie de l'être assurée ontologiquement, s'appliquent mieux à Dieu qu'aux créatures (ce qui n'est pas le cas pour le 'rocher'). Parce que toute bonté et sagesse proviennent du Créateur qu'est Dieu, il est plus approprié d'appeler Dieu bon et sage, que de le dire par exemple d'êtres humains ou d'animaux. Dans la procession de l'être, ces derniers ne peuvent jamais posséder la bonté ou la sagesse au même degré que la source de laquelle ils procèdent. Toutefois, dans la perspective de la création, Thomas d'Aquin réaffirme que dire à propos de Dieu qu'il est bon et sage n'implique pas un savoir touchant à l'essence de Dieu. En effet, du fait de la différence ontologique entre Créateur et création, ce n'est qu'à partir de la création, c'est-à-dire à partir de nos expériences humaines, que nous savons ce que sont la bonté et la sagesse ${ }^{25}$. De nos jours, une telle fondation ontologique du discours chrétien sur Dieu est problématisée, et le statut cognitif spécial de l'analogie avec elle. Par ailleurs, le mode analogique peut être clarifié par une approche sémantico-cognitive de la métaphore ou, dans ces cas, de la métonymie. DEU EST BON et DIEU EST SAGE sont des métaphores (ou plutôt, des schèmes métonymiques) qui superposent la conceptualité du domaine de la BONTÉ et de la SAGESSE au domaine de DIEU, structurant ainsi les expériences, les interprétations - tout comme le comportement correspondant - de la relation du croyant à Dieu et à sa création. C'est vrai aussi pour DIEU EST PÈRE. Toujours selon Thomas d'Aquin, cette affirmation basée sur une évidence scripturaire est aussi une analogie, structurant métaphoriquement le domaine de DEU en termes de paternité. Plus généralement, l'on pourrait montrer que, outre un tel ressaisissement de la catégorie de l'analogie, la redéfinition de la cognitivité réévalue

${ }^{25}$ Summa Theologiae, Ia, O. 13, art. 3: «Nous l'avons dit, nous connaissons Dieu au moyen des perfections qui procèdent de lui dans les créatures. Or notre intellect appréhende ces perfections telles qu'elles sont dans les créatures, et selon la façon dont il les appréhende, il les signifie par des noms; toutefois, dans les noms que nous appliquons à Dieu, deux choses sont à considérer: les perfections mêmes signifiées par ces mots, comme la bonté, la vie, etc., et la manière dont elles sont signifiées. Quant à ce que signifient ces noms, ils conviennent à Dieu en propre, et plus encore qu'aux créatures, et en priorité. Mais quant à la manière de signifier, ces mêmes noms ne s'appliquent plus proprement à Dieu, car leur mode de signification mêmes ni signification Théologique, A. RAULIN et A.-M. RoguET (eds.), Paris, 1999², t. 1, p. 239. 
de manière positive le rôle cognitif traditionnellement sous-estimé du mode métaphorique du discours sur Dieu (par exemple, DIEU EST UN ROCHER).

En outre, la présomption apophatique que Thomas d'Aquin a préservée dans le mode analogique - à savoir que du point de vue de la création le discours sur Dieu n'est pas apte à signifier Dieu - est sauvegardée et même radicalisée par une approche sémantico-cognitive. Souligner la non-autonomie sémantique de DIEU équivaut à critiquer toute lecture littérale d'une conception de Dieu. De ce point de vue, sur le plan théologique, toute conceptualisation de DIEU devrait, d'une façon ou d'une autre, être accompagnée de la conscience que dans l'acte de la superposition métaphorique, la cible (DIEU) se dérobe constamment D\&U. La connaissance que nous avons de Dieu est structurée à partir de nos expériences et de nos contextes socioculturels, reflétés par cette connaissance. Ainsi, nos concepts de Dieu établis et conventionnels ne reflètent pas un Dieu existant comme tel et qui serait à découvrir dans son essence. C'est également à cause de nouvelles expériences et de changements dans les contextes socioculturels que nos conceptualisations traditionnelles de Dieu sont mises sous pression et commencent à se transformer. Le discours sur Dieu et, de manière plus générale, la tradition religieuse sont donc impliqués dans un processus continuel de recontextualisation.

\section{Critique de la tradition: mécanismes sous-jacents aux processus de 'littéralisation'}

À la lumière de ce qui a été dit plus haut, il est clair que la structure théorique de la linguistique cognitive fournit aux théologiens une intelligence du mécanisme sous-jacent au processus toujours menaçant de la 'littéralisation' ou objectivation des métaphores religieuses. Le comparatif est donc considéré à tort comme du constatif. L'imaginaire religieux est tenu pour du langage descriptif dont le statut cognitif est défini en termes de correspondance. En fait, au cours de l'histoire religieuse, la chrétienté a souvent été confrontée à la tentation d'oublier les superpositions métaphoriques structurelles sousjacentes au discours religieux. De ce point de vue, la linguistique cognitive attire l'attention sur la fondation expérimentale de notre système de connaissance, et montre que, dans l'objectivation de métaphores, la structure du domaine-source de la superposition est acceptée comme la structure essentielle du domaine-cible ${ }^{26}$. On peut trouver des illustrations importantes dans l'ancienne vision du monde prémoderne et chrétienne de 'La grande chaîne de l'être': Dieu au ciel étant le point de départ, on descend progressivement, via les anges, jusqu'aux êtres humains et aux plantes pour arriver enfin à la matière inanimée ${ }^{27}$. C'est un modèle culturel impliquant des métaphores structurantes, et qui permet de comprendre les attributs et les comportements d'êtres supérieurs en termes d'attributs ou d'essences d'êtres inférieurs ${ }^{28}$. Dans ce contexte, l'on pourrait attirer l'attention sur l'amalgame de l'imagination religieuse et de la cosmologie (par exemple, les anges étant les causes des mouvements planétaires), et ainsi sur la spatialisation et la temporalisation de l'eschatologie chrétienne (la doctrine des fins dernières): suivant l'idée que LE BIEN EST EN AMONT et LE MAL EST EN AVAL, le ciel est situé au-dessus de nous, et l'enfer en-dessous.

En même temps, nous devons reconnaître que malgré la faillite de la vision du monde prémoderne, la GRANDE CHAÎNE DE L'ÊTRE est toujours très active sur le plan métaphorique «comme un modèle culturel inconscient et contemporain, indispensable à la compréhension de nous-mêmes, de notre monde et de notre langage ${ }^{29}-$ et, plus particulièrement, du langage religieux. Son socle expérimental permet toujours de comprendre par exemple - sans pour autant s'abandonner à l'objectivation - la formule du credo concernant la descente aux enfers, la résurrection des morts et l'ascension au ciel qui réalise ENDORMI, MORT EST EN AVAL, et ÉVEILLÉ, VIVANT EST EN AMONT.

${ }^{26}$ C'est dans ce contexte, du moins pour les philosophes et les théologiens, qu'il vaut la peine de considérer l'usage que fait Lakoff du mot 'ontologique'. En effet, pour lui, 'ontologique' ne renvoie pas à l'essence objective des choses, mais soupour lui, 'ontologique' ne renvoie pas à l'essence objective des choses, mais sou-
ligne un ensemble de correspondances structurelles dans notre expérience des domaines-cible et des domaines-source de la superposition métaphorique (par exemple G. LAKOFF, The Contemporary Theory of Metaphor, dans A. ORTONY (ed.), Metaphor and Thought, Cambridge, $1993^{2}$, p. 207).

${ }^{27}$ La GRANDE CHAÎNE DE L'ÊTRE est définie comme «un modèle culturel qui concerne des manières d'être et leurs propriétés, et qui les situe sur une échelle verticale avec des êtres 'supérieurs' et des propriétés correspondantes plus élevés que les êtres 'inférieurs' et leurs propriétés. [...] La GRANDE CHAÎNE DE L'ÊTRE est une les êtres 'inférieurs' et leurs propriétés. [... La GRANDE CHAÎNE DE L'ÊTRE est une
échelle de formes d'être - homme, animal, plante, objet inanimé - et, par conséquent, une échelle de propriétés qui les caractérisent - raison, comportement instinctif, fonction biologique, attributs physiques, etc.» (G. LAKOFF et M. TURNER, More than Cool Reason [n. 20], p. 166-167)

${ }^{28}$ Ibid., p. 160 et sv.

29 Ibid., p. 167. 
Les mêmes processus d'objectivation sont pourtant également à l'œuvre dans ce qu'on appelle notre discours spéculatif ou conceptuel sur Dieu. Souvent, les théologiens ont tendance à oublier que même les doctrines théologiques très fondamentales DIEU EST UNE PERSONNE et DEU EST AMOUR sont métaphoriques. On pourrait arguer que les tentatives de certains théologiens pour rester fidèles à la doctrine de la toute-puissance de Dieu - ce qui requiert des efforts herméneutiques énormes - sont à situer dans le cadre d'une objectivation d'une caractéristique inhérente aux métaphores du LEADERSHIP, qui impliquent l'autorité: DIEU EST ROI/EMPEREUR, DIEU EST LE SEIGNEUR DES ARMÉES/FOULES, DIEU EST JUGE ${ }^{30}$.

Il semble qu'on rencontre beaucoup de résistance et d'ambiguïté, à cause des conceptions traditionnelles de la métaphore, si l'on qualifie le discours théologique de métaphorique, quoique ce soit précisément là - au moins en utilisant la linguistique cognitive - que la conscience critique herméneutique de la religion peut opérer à plein.

\section{Développement de la tradition: le principe d'économie}

La linguistique cognitive permet aussi à la théologie de clarifier comment, à travers 1'histoire, beaucoup de significations différentes ont été exprimées avec un même vocabulaire (i.e. le principe d'économie). À la lumière des superpositions métaphoriques impliquées dans le savoir religieux, on peut voir en effet comment les domainessource étaient et sont situés dans un contexte spécifique, et comment ces domaines ont été affectés par des changements du contexte. De tels changements peuvent être dus à des événements historiques et à des variations culturelles internes, mais ils sont également provoqués par la simple traduction de textes, dès lors que des concepts ne sont pas structurés en termes du même domaine-source dans la langue originale et dans leurs traductions. Les deux métaphores DIEU EST UNE PERSONNE et DIEU EST PÈRE, par exemple, représentent à l'époque actuelle des structurations conceptuelles qui diffèrent des structurations antérieures à la philosophie personnaliste et à la critique féministe du patriarcat.

${ }^{30}$ Pour ces métaphores du LEADERSHIP, voir L. BoEve et K. FEyAERTs, Religious Metaphors in a Postmodern Context (n. 3), p. 20-23.
Une meilleure intelligence du principe d'économie soulève évidemment des questions à propos des présuppositions de continuité et/ou de discontinuité dans le développement de la critique. La continuité est-elle plus qu'une simple affaire d'emploi du même vocabulaire? Et - ouvrant ainsi la perspective de notre thèse suivante - la discontinuité doit-elle être mise sur pied d'égalité avec l'introduction d'un nouveau vocabulaire (ou la redéfinition radicale de mots et d'expressions existants)?

\section{Développement de la tradition: recontextualisation}

La question de savoir pourquoi la tradition chrétienne a changé recevra également une réponse, du moins partielle, si l'on considère ce que dit la linguistique sur la façon dont la tradition se développe, dont elle crée un vocabulaire nouveau, des structures réflexives nouvelles, etc. Comme il a été dit plus haut, une interrelation dynamique, déterminée contextuellement entre une charpente (tradition) interprétative établie et de nouvelles expériences, peut être découverte à cause de changements dans la tradition religieuse. On trouve un écho de ce fait dans la présupposition sémantico-cognitive que les métaphores, et donc les changements dans les métaphores, sont motivés aussi du fait de leur fondement expérimental. À cet égard, il vaut la peine de réévaluer les nouveaux modèles de Dieu proposés par Sally McFague, et qui prennent appui sur des sensibilités contemporaines (DIEU EST MÈRE, DIEU EST UN AMANT, DIEU EST UN AMI).

Un élément majeur dans le développement de la tradition, et qui peut être exploité par la linguistique cognitive dans un but théologique, est la traduction du discours religieux, d'une langue à une autre. Un changement théologique pertinent dans la compréhension métaphorique de la relation de Dieu avec les êtres humains a été examiné par Yri $^{31}$. Il a démontré que l'activité de Dieu dans la Bible hébraïque était surtout structurée en termes d'assistance politico-militaire lors de conflits et de situations d'oppression, tandis que, dans la Septante, ce modèle militaire était remplacé par un équivalent médical. DIEU EST UN SAUVEUR implique alors des superpositions engendrées par le domaine

${ }^{31}$ K.M. YRI, Father Taught Me How to Cry, But Now I Have Forgotten. The Semantics of Religious Concepts With an Emphasis on Meaning, Interpretation, and Translatability (coll. Acta humaniora, 29), Oslo, 1998. 
de GARDER EN BONNE CONDITION, PRÉSERVER OU RENDRE LA SANTÉ, PRÉSERVER OU RENDRE LA VIE. Au niveau théologique, ceci implique une acception très différente de la relation salvatrice de Dieu avec l'humanité, qui, en effet, se spiritualise, s'individualise, se privatise même. Le salut n'est plus socio-politique, il se centre sur la santé spirituelle; Dieu n'est plus d'emblée perçu comme le leader de l'aventure historico-politique du peuple élu, il est plutôt comme le médecin guérissant un patient.

C'est contre une telle individualisation, une telle spiritualisation ou privatisation du salut que les théologies politiques européennes et les théologies de la libération latino-américaines ont réagi. En se référant aux récits libérateurs de l'exode dans l'Ancien Testament, elles ont changé la métaphore DIEU/JÉSUS EST UN SAUVEUR en DIEU/JÉSUS EST UN LIBÉRATEUR. Pour des raisons contextuelles (pauvreté massive causée par l'injustice structurelle), les théologies politiques et de la libération ont souligné l'impact socio-politique et historique de l'action salvatrice de Dieu, et critiqué des conceptions plus exclusivement spirituelles du salut, telles que la santé spirituelle et la vie éternelle. En effet, ces dernières semblent ne pas àvoir de conséquences pratiques pour le bien-être personnel et social ici et maintenant (LE SALUT COMME LA PRÉSERVATION DE LA VIE contre LE SALUT COMME LA LIBÉRATION DE LA PRISON, DE L'ESCLAVAGE, DE L'OPPRESSION, DE LA PAUVRETÉ). Quand on considère plus attentivement la critique adressée à la théologie de la libération - critique formulée entre autres par le cardinal Ratzinger - on remarquera qu'elle se concentre précisément sur l'emploi du motif de l'exode (incluant ainsi le glissement métaphorique): elle reproche aux théologiens de la libération d'oublier l'universalisation et la radicalisation de l'événement de l'exode dans le baptême chrétien ${ }^{32}$.

Enfin, un dernier exemple peut étayer l'idée que c'est surtout dans l'analyse de la tradition que la linguistique cognitive offre des structures utiles pour la théologie: à travers son analyse des superpositions métaphoriques, elle clarifie des glissements ou des discontinuités dans le développement théologique. Dans Lumen Gentium, la constitution dogmatique sur l'Église du Concile Vatican II, les pères conciliaires ont opté pour une nouvelle métaphore ecclésiale:

32 J. RATZINGER, Jesus Christus heute, in Internationale Katholische Zeitschrift Communio, t. 19, 1990, p. 56-70.
'1'Église est le peuple-pèlerin de Dieu', à côté de la métaphore plus traditionnelle 'l'Église est le corps du Christ', dans le but de comprendre la nature de l'Église d'une façon plus humaine, communautaire et historique. La métaphore L'ÉGLISE EST LE CORPS DU CHRIST ( $L G 7)$ se prête à une ecclésiologie statique et hiérarchique (impliquant en réalité les structures de LA GRANDE CHAÎNE DE L'ÊTRE ${ }^{33}$ ). L'ÉGLISE EST LE PEUPLE DE DIEU ( $L G 9$ ), par contre, accentue le pèlerinage séculier de l'Église vers le royaume de Dieu, et invoque mieux la dynamique impliquée dans LA VIE EST UN VOYAGE - ou, mutatis mutandis, ÊTRE ÉGLISE EST UN VOYAGE.

5. Une approche interdisciplinaire asymétrique: 'linguistica ancilla theologiae'

L'analyse des concepts religieux suivant la théorie de la métaphore sémantico-cognitive ne constitue pas l'objectif ultime de la théologie. L'usage possible de la linguistique cognitive doit toujours être motivé, légitimé, et donc déterminé ou limité théologiquement. La théologie ne devrait pas être cantonnée de force à 1'intérieur du point de vue qu'a un simple observateur sur la langue ou sur la réalité de la religion. Au contraire, prenant explicitement appui sur une tradition et une communauté religieuse particulière, elle devrait présenter le point de vue réflexif d'un participant. La théologie comme fides quaerens intellectum ne se contente donc pas de la linguistique cognitive. Pour la théologie, la linguistique cognitive n'est qu'un instrument qui est à son service, une occasion possible de ressaisir l'intellectus de la foi chrétienne dans notre contexte contemporain. Ainsi, l'aspiration à la cognitivité en théologie ne concerne-t-elle plus la fondation ou la légitimation des prétentions chrétiennes à la vérité. Mais elle peut établir la plausibilité et l'intelligence de la compréhension chrétienne en révélant les structures conceptuelles qui y sont impliquées. Ce point peut être illustré par d'autres exemples et réflexions.

Premièrement, quoiqu'elle soit consciente de son incapacité à saisir Dieu et son salut, la théologie enracinée dans la foi d'une

33 Voir G. LAKOFF et M. TURNER, More than Cool Reason (n. 20), p. 211, qui signalent les conséquences éthiques, sociales, politiques et religieuses de la réalisation de la GRANDE CHAînE. 
communauté croyante reste intéressée - d'une autre manière donc que la linguistique cognitive - par la réalité objective de ce qu'elle professe: Dieu et son salut. Pour la linguistique cognitive, que Dieu existe ou non, seule est importante la cognitivité fondée expérimentalement, quoique finalement toujours intérieure, impliquée dans le langage religieux et dans la conceptualité religieuse. En tant que concept, DIEU n'engendre pas plus de difficultés que d'autres concepts abstraits comme TEMPS, AMOUR, IDÉE, etc. De ce point de vue, la linguistique cognitive s'en tient à un agnosticisme ou un athéisme méthodologique. Bien que leurs prétentions à la vérité soient mises en cause dans notre contexte post-moderne, les théologiens continueront quand même à poser la question de la vérité, et c'est seulement dans cette perspective que la linguistique cognitive peut intervenir. Aussi, lorsque les théologiens feront usage de la linguistique cognitive, ils devront compléter 1'approche de la cognitivité par ce que nous avons appelé une cognitivité 'hyperphatique', la cognitivité du langage religieux étant saisie en une structure référentielle irréparablement fracturée ${ }^{34}$.

Deuxièmement, 1'emploi théologique de la linguistique cognitive n'évacue pas le besoin d'interprétation théologique; au contraire, il le renforce. En tant que sémanticien cognitif, Olaf Jäkel a examiné la métaphore du VOYAGE dans un aperçu biblique d'une vie vouée à la foi. MENER UNE VIE BONNE équivaut alors à FAIRE UN VOYAGE SUR LE CHEMIN DE DIEU (ce qui implique par exemple PÉCHER EST DÉVIER, SE REPENTIR EST SE RETOURNER); MENER UNE VIE IMMORALE, par contre, est PRENDRE LE CHEMIN DU MAL ${ }^{35}$. En s'appuyant sur des preuves scripturaires, Jäkel distingue en outre deux chemins (LE CHEMIN DE DIEU EST DROIT; LES CHEMINS DU MAL SONT SINUEUX, PLEINS D'OBSTACLES), deUX sortes de voyageurs, et deux façons dont Dieu s'occupe de ces derniers (DIEU EST LE GUIDE; DIEU OBSTRUE LES CHEMINS DES MÉCHANTS). Jusqu'ici, cette analyse confirme, pour ainsi dire, l'importance de la métaphore du VOYAGE dans la structuration de notre compréhension de la VIE (ici la VIE RELIGEUSE). Or, les choses se compliquent pour Jäkel quand il analyse les mêmes structures métaphoriques dans le Nouveau Testament, quand l'intelligence qu'a le croyant de JÉsus lui-même est

${ }^{34}$ Pour un compte rendu de la référentialité du langage théologique, voir J.M. SoskICE, Metaphor and Religious Language, Oxford, 1985.

${ }^{35}$ O. JÄKEL, «How can mortal man understand the road he travels? » Prospects and Problems of the Cognitive Approach to Religious Metaphor (LAUD-Preprint: Series A, 418), Duisburg, 1997. structurée au moyen de la métaphore du VOYAGE, ce qui transforme JÉSUS non seulement en guide, mais également en chemin, en porte: «Je suis le chemin, la vérité et la vie» (Jn 14,6). Le commentaire de Jäkel dit: «indubitablement, on sent une dissonance dans ces exemples. [...] Sans doute, ces affirmations soulignent-elles l'importance de Jésus pour le croyant. Mais peut-être la dissonance conceptuelle est-elle voulue et la violation de la cohérence métaphorique ordinaire fait-elle allusion au caractère 'métaphysique', et 'surnaturel' de toute l'entreprise de la vie religieuse». Jäkel ajoute à juste titre que c'est une question à résoudre par le théologien ${ }^{36}$. En tant que théologien, je doute que les termes 'métaphysique' et 'surnaturel' jettent plus de lumière sur la dissonance analysée. Pourtant, la dissonance mentionnée éclaire de façon particulière l'affirmation théologique selon laquelle le message chrétien ne consiste pas seulement en la proclamation du Royaume à venir, ainsi que Jésus l'a professé, mais également en Jésus-Christ lui-même, considéré après l'expérience de sa résurrection, comme médiateur du salut divin. En effet, la spécificité de l'Évangile chrétien est précisément l'indissoluble relation entre le message et le messager.

En guise de troisième illustration, je mettrai en évidence la relation inhérente, reconnue théologiquement, entre la cognitivité du langage religieux et la conscience critique religieuse. La cognitivité hyperphatique par exemple, s'active, pour ainsi dire, quand à partir de la cible religieuse une critique inverse est effectuée vers le domaine-source original du langage religieux. Quand on comprend DIEU comme ROI, ou comme AMI, une conscience critique motivée religieusement devient opérante et se meut vers les domaines-source de la royauté et de l'amitié. Le fait que certains qualifient DIEU également de MÈRE, non seulement en dit long sur les glissements dans notre contexte, mais installe en même temps une conscience critique motivée religieusement vis-à-vis des sensibilités et structures patriarcales et sociales.

\section{CONCLUSION}

Dans cet essai nous avons renchéri sur l'attention considérable que la catégorie de la métaphore reçoit déjà en linguistique et,

${ }^{36}$ Ibid., p. 18. 
depuis, dans les épistémologies philosophiques et théologiques (G. Lakoff, S. McFague, P. Ricœur, J.M. Soskice, J.P. Van Noppen et al.). La plupart des théologiens ne seront pas surpris par nos constatations, mais celles-ci sont intégrées dans un vocabulaire spécifique et dans une structure réflexive. C'est ce qui permettra à la théologie de poser quelques questions et de scruter quelques positions traditionnelles à nouveaux frais. En effet, ma tentative d'analyse de la pertinence théologique de la théorie sémantico-cognitive de la métaphore est un appel à un examen plus développé, non seulement de son utilité théologique, mais également de quelques questions théologiques spécifiques comme le statut du langage religieux, la nature des prétentions théologiques à la vérité, la continuité et la discontinuité dans le développement de la tradition etc. J'espère que cet article contribuera à une meilleure compréhension de la pertinence d'une telle tentative, non seulement pour les linguistes et les biblistes, qui semblent déjà convaincus, mais également pour les théologiens systématiques ${ }^{37}$.

\section{B - 3010 Kessel Lo, Platte Losstraat 592.}

Lieven BOEVE, Professeur aux Facultés de théologie de la K.U.Leuven et de l'U.C.L.
Résumé - Lorsqu'il laisse interagir l'expérience contextuelle et l'interprétation traditionnelle, le langage religieux apporte du neuf. L'auteur se demande si la «linguistique cognitive» offre certaines pistes de réflexion et certains modèles utiles à la recontextualisation de l'épistémologie théologique dans la situation actuelle de la postmodernité. La sémantique cognitive présente la théologie comme une approche de la réalité dans un cadre conceptuel fondamental et non dans un rapport prédéterminé entre l'ordre logique et l'ordre ontologique. La théorie de la métaphore est ici particulièrement suggestive. Elle montre que certains concepts - la plupart du temps abstraits, comme AMOUR et DIEU - sont essentiellement structurés métaphoriquement, parce qu'ils sont sémantiquement non autonomes. En cinq remarques conclusives, l'auteur montre comment l'approche interdisciplinaire de la linguistique cognitive peut servir la théologie d'aujourd'hui, tout en manifestant ses limites.

${ }^{37}$ Cet article est une version modifiée d'une présentation que j'ai faite au colloque Meaning, Metaphor \& Religion: Cognitive Semantics and the Bible, à Leuven, 6-8 juillet 1998. La traduction est de Geert Misotten, K.U.Leuven.
Summary - In the interaction of contextual experience and traditional interpretation, religious language originates and shifts. In this contribution the author investigates whether cognitive linguistics can offer some interesting ideas and thought patterns for a recontextualisation of theological epistemology in our current postmodern condition. Cognitive semantics teaches theology that cognitivity lies in the conceptual framework underlying our approach of reality, and not in a presumed determinate relationship between the logical and the ontological order. Of particular interest is its theory of metaphor, showing that many concepts - mostly abstract ones, like LOVE, GOD - are essentially structured metaphorically, because they are semantically non-autonomous. In five concluding observations, the author sketches how an interdisciplinary encounter with cognitive linguistics can serve theology today, as well as revealing its limitations. 